\title{
High-fat diet and colorectal cancer: myths and facts
}

\author{
Sheelarani Karunanithi*,1,2 \& Liraz Levi ${ }^{1}$ \\ ${ }^{1}$ Case Comprehensive Cancer Center, Case Western Reserve University, Cleveland, OH 44106, USA \\ ${ }^{2}$ Department of Pathology, Case Western Reserve University, Cleveland, OH 44106, USA \\ * Author for correspondence: srk115@case.edu
}

"Furthermore, there is mounting epidemiological data on the possibility of HFD increasing colon cancer risk and recurrence which cannot be completely overlooked due to inconsistencies and lack of mechanistic evidence."

First draft submitted: 27 October 2017; Accepted for publication: 14 November 2017; Published online: 7 February 2018

Keywords: colon cancer $\bullet$ diet $\bullet$ high-fat $\bullet$ obesity $\bullet$ stemness

Colorectal cancer affects over 100,000 patients yearly and is the second leading cause of cancer-related deaths in the USA. The incidence and survival rates of colorectal cancer patients have improved steadily in the past decade primarily due to early diagnosis and awareness [1]. Several genetic and epigenetic causes of colon cancer have been identified and their mechanisms explained, such as that for Wnt pathway genes, TGF $, T P 53, K R A S$ and others. Data from epidemiological studies on the influence of patients' lifestyle and dietary habits indicate that these factors affect the risk of developing colon cancer [2]. Understanding the contribution of each of these environmental factors to the risk of colon cancer and elucidating the molecular mechanisms underlying their function is crucial for prevention and treatment of the disease.

In the recent decades, increased prevalence of diet- and overweight-related morbidities have categorized obesity as a growing epidemic [3]. Obesity prevalence is accompanied by an increase in associated comorbidities such as cardiovascular disease, stroke, nonalcoholic fatty liver disease and even cancer [4]. Indeed, cancer statistics from Centers for Disease Control and Prevention show a 7\% increase in obesity-related cancers (pancreatic, breast, liver and others) during the years 2005-2014, while the incidence of other cancers have decreased by $13 \%$ during the same time period [5]. Ning et al. performed a meta-analysis to show that increase in BMI caused a proportional increase in colon cancer risk [6].

Dietary factors including high fat, high protein, low vitamin D and low calcium are thought to contribute to $80 \%$ of colon cancer cases [7]. The effect of some risk factors (red meats, calcium) on colon carcinogenesis have been fairly established using several animal models and population-based studies; however the link between other components (fat, vitamin D, folate) and colon cancer remains unclear [8]. An elegant study by Newmark et al. show that mice fed a western diet (high fat, low vitamin D, calcium and folate) had induced colonic tumors in their intestines [9]. Although this was one of the first reports showing a direct connection between western diet and development of colon cancer (in the absence of any carcinogen), this study is limited in interpreting the exact effect of each of the dietary components. High fat containing diet (HFD) was identified to influence colon cancer risk as early as the 1960s mostly based on the geographical distribution of colon cancer epidemiology. While several animal and cell culture studies point to a direct role of dietary fats in increasing colonic cell proliferation and associated risks [10-12], interpretations from population studies are more complex and inconsistent. For example, a retrospective study showed no risk association between high and low animal fat intake in a meta-analysis [13].

Although the association between HFD and colon cancer risk has been known for several years, the exact mechanisms underlying the HFD-induced colon cancer risk and recurrence have remained unclear. HFD can increase colon cancer incidence either directly or via high-fat-induced obesity. Obesity driven changes such as chronic low-grade inflammation, insulin resistance, fat-derived hormonal imbalance and gut microbiota are all known to promote colon cancer incidence [14]. Studies that test the direct effects of HFD on colon cancer risk have 
been sparse and inconsistent. Diets high in fat content can increase bile acid deposition into the colon, presumably for fat emulsification and in turn, bile acids, such as deoxycholic acid, that are known to be carcinogenic can increase colon cancer propensity [15]. Dietary fats were recently shown to promote $\mathrm{Lgr}^{+}{ }^{+}$intestinal stem cell pool and their tumorigenicity via PPAR $\delta$-mediated pathway in a model of HFD-induced obesity in mice [12]. This study also highlighted the possibility that dietary fats per se can induce this phenotype using ex vivo models. It was recently demonstrated that HFD can induce colon carcinogenesis and LGR5 expression in a xenograft model of colon cancer independent of obesity through a RBP4-STRA6 signaling pathway [16]. The specific role of different types of fats in promoting colon cancer risk are also fairly confusing. Polyunsaturated fats $(n-3)$ are shown to be protective against colon cancer due to their anti-inflammatory properties [17]. Saturated fats, on the other hand, can promote colon cancer risk [18] while also providing a protective role by increasing mucin production in some animal models of colon cancer [19].

The challenge in tracing the exact role of dietary fats in vivo can partly be attributed to the lack of addressing the difference in their caloric value and carcinogenicity, and the difficulty to distinguish between effects attributed by obesity from those of HFD per se. Some of these challenges may be overcome by utilizing mice strains (BALB/c, athymic nude, $\mathrm{FVB} / \mathrm{N}$ ) that are resistant to diet-induced obesity and can be used to investigate HFD-induced colon carcinogenesis. These models can further be used in studies testing the contribution of different types of dietary fats to better understand their individual properties. Population studies are more complicated due to missing data in chart reviews and lack of reporting and compliance by study participants in prospective studies. Successful data mining approaches to assess HFD to colon cancer risk would entail strict rules on clustering data based on dietary factors, dietary habits and geographical location of study participants, and minimizing the projections from combinatorial effects of dietary factors. Several studies related to insulin resistance, diabetes and obesity also require similar data input and have begun to incorporate smartphone application-based studies to collect data with improved success [20]. A probable way to overcome limitations in studying the HFD-colon cancer incidence may be to repurpose some of these diabetic and obesity models and applications for long-term risk assessment of colorectal cancer. Another interesting possibility is to develop a publicly available global repository and analysis software that encompasses available data on the effect of dietary factors and obesity to various comorbidities such as diabetes, cancer and others. Such a web-based organizer may revolutionize the possible analyses on existing as well as new data in ways comparable to tools such as Oncomine that transformed the understanding of gene expression and cancers.

In summary, there is a clear association linking obesity to colon cancer risk. Furthermore, there is mounting epidemiological data on the possibility of HFD increasing colon cancer risk and recurrence which cannot be completely overlooked due to inconsistencies and lack of mechanistic evidence. More collaborative, multidisease, multicenter and innovative data mining approaches may soon overcome the limitations seen in the past five decades and improve colon cancer prevention and management.

\section{Financial \& competing interests disclosure}

The authors have no relevant affiliations or financial involvement with any organization or entity with a financial interest in or financial conflict with the subject matter or materials discussed in the manuscript. This includes employment, consultancies, honoraria, stock ownership or options, expert testimony, grants or patents received or pending, or royalties.

No writing assistance was utilized in the production of this manuscript.

\section{References}

Papers of special note have been highlighted as: $\bullet$ of interest; $\bullet \bullet$ of considerable interest

1. Howlader N, Noone AM, Krapcho M et al. SEER Cancer Statistics Review, 1975-2013. National Cancer Institute, Bethesda, MD, USA (2016). http://seer.cancer.gov/csr/1975_2013/

2. Walther A, Johnstone E, Swanton C, Midgley R, Tomlinson I, Kerr D. Genetic prognostic and predictive markers in colorectal cancer. Nat. Rev. Cancer 9(7), 489-499 (2009).

3. Caballero B. The global epidemic of obesity: an overview. Epidemiol. Rev. 29(1), 1-5 (2007).

4. Goodwin PJ, Stambolic V. Impact of the obesity epidemic on cancer. Annu. Rev. Med. 66(1), 281-296 (2015).

- Details the known connections between obesity and different types of cancers.

5. Steele CB, Thomas CC, Henley SJ et al. Vital signs: trends in incidence of cancers associated with overweight and obesity - United States, 2005-2014. MMWR Morb. Mortal. Wkly Rep. (66), 1052-1058 (2017). 
6. Ning Y, Wang L, Giovannucci EL. A quantitative analysis of body mass index and colorectal cancer: findings from 56 observational studies. Obes. Rev. 11(1), 19-30 (2010).

7. Nyström M, Mutanen M. Diet and epigenetics in colon cancer. World J. Gastroenterol. 15(3), 257-263 (2009).

8. Vargas AJ, Thompson PA. Diet and nutrient factors in colorectal cancer risk. Nutr. Clin. Pract. 27(5), 613-623 (2012).

9. Newmark HL, Yang K, Lipkin M et al. A Western-style diet induces benign and malignant neoplasms in the colon of normal C57Bl/6 mice. Carcinogenesis 22(11), 1871-1875 (2001).

-• Shows the development of colon neoplasm in wild-type mice fed a western diet.

10. Mao J, Hu X, Xiao Y et al. Overnutrition stimulates intestinal epithelium proliferation through $\beta$-catenin signaling in obese mice. Diabetes 62(11), 3736-3746 (2013).

11. Yilmaz $\mathrm{OH}$, Katajisto P, Lamming DW et al. mTORC1 in the Paneth cell niche couples intestinal stem-cell function to calorie intake. Nature 486(7404), 490-495 (2012).

-. Highlights the effect of calorie restriction on intestinal stem cell function.

12. Beyaz S, Mana MD, Roper J et al. High-fat diet enhances stemness and tumorigenicity of intestinal progenitors. Nature 531(7592), 53-58 (2016).

-• Demonstrates that high-fat diet increases intestinal stemness.

13. Alexander DD, Cushing CA, Lowe KA, Sceurman B, Roberts MA. Meta-analysis of animal fat or animal protein intake and colorectal cancer. Am. J. Clin. Nutr. 89(5), 1402-1409 (2009).

14. O’Keefe SJD. Diet, microorganisms and their metabolites, and colon cancer. Nat. Rev. Gastroenterol. Hepatol. 13(12), 691-706 (2016).

- Impactful review of the current literature on gut microbiota and diet-associated colon cancer.

15. Bernstein C, Holubec H, Bhattacharyya AK et al. Carcinogenicity of deoxycholate, a secondary bile acid. Arch. Toxicol. 85(8), 863-871 (2011).

16. Karunanithi S, Levi L, DeVecchio J et al. RBP4-STRA6 pathway drives cancer stem cell maintenance and mediates high-fat diet-induced colon carcinogenesis. Stem Cell Reports 9(2), 438-450 (2017).

-• Links vitamin A signaling to high-fat diet-induced colon cancer.

17. Hodge AM, Williamson EJ, Bassett JK, Macinnis RJ, Giles GG, English DR. Dietary and biomarker estimates of fatty acids and risk of colorectal cancer. Int. J. Cancer 137(5), 1224-1234 (2015).

18. Willett WC, Stampfer MJ, Colditz GA, Rosner BA, Speizer FE. Relation of meat, fat and fiber intake to the risk of colon cancer in a prospective study among women. N. Engl. J. Med. 323(24), 1664-1672 (1990).

19. Enos RT, Velázquez KT, McClellan JL et al. High-fat diets rich in saturated fat protect against azoxymethane/dextran sulfate sodium-induced colon cancer. Am. J. Physiol. Gastrointest. Liver Physiol. 310(11), G906-G919 (2016).

- Discusses a potential benefit of saturated fat on colon cancer.

20. Gill S, Panda S. A smartphone app reveals erratic diurnal eating patterns in humans that can be modulated for health benefits. Cell Metab. 22(5), 789-798 (2015). 
